\title{
ON THE ANALOGUE FOR DIFFERENTIAL EQUATIONS OF THE HILBERT-NETTO THEOREM
}

\author{
RICHARD COHN
}

If

$$
F_{1}, \cdots, F_{r}
$$

is a finite system of differential polynomials in the unknowns $y_{1}, \cdots, y_{n}$, and if $G$ is a differential polynomial which is annulled by every solution of the system (1), some power $G^{p}$ of $G$ is a linear combination of the $F_{i}$ and their derivatives of various orders, with differential polynomials for coefficients. This analogue of the HilbertNetto theorem was proved by J. F. Ritt ${ }^{1}$ for forms with meromorphic coefficients, and by H. W. Raudenbush ${ }^{2}$ for the case of coefficients belonging to an abstract differential field. In these proofs it is shown that the denial of the existence of the exponent $p$, above, of $G$ leads to a contradiction; no constructive method for obtaining admissible values of $p$ is given. The object of the present note is to present a new proof of the analogue, for the case of meromorphic coefficients, which is entirely constructive and produces a definite $G^{p}$ as described above.

Our proof will be based on the considerations in Chapters $\mathrm{V}$ and VII of A.D.E. In Chapter VII, the problem of obtaining $G$ is reduced to the problem of determining unity as a linear combination of the $F_{i}$ in (1) and their derivatives, in the case in which (1) has no solutions. In Chapter $\mathrm{V}$ it is shown how to decide, in a finite number of steps, whether or not (1) has solutions. Our problem thus assumes the following form: Given that (1) has no solutions, it is required to express unity as a linear combination of the $F_{i}$ and their derivatives.

We assume that (1) has no solutions and proceed to examine the algorithm developed in $\$ \$ 65-67$ of A.D.E. Adjoining to (1) a finite number of linear combinations of the $F_{i}$ and their derivatives, we obtain a system $\Sigma$, devoid of solutions, with a basic set

$$
A_{1}, \cdots, A_{q}
$$

which has the property that the remainder of every form in $\Sigma$ with respect to (2) is zero. If (2) consists of a single form $A$ which is an

${ }^{1}$ Ritt, J. F., Differential Equations from the Algebraic Standpoint, chap. 7, referred to below as A.D.E. American Mathematical Society Colloquium Publications, vol. 14, 1932.

${ }^{2}$ Raudenbush, H. W., Ideal theory and algebraic differential equations, Transactions of this Society, vol. 36 (1934), pp. 361-368. 
element of $\mathcal{F}$, the coefficient field of our forms, we secure immediately a representation of the type desired for unity. Let us suppose that this is not the case. Then (2), considered as a set of simple forms, cannot be a basic set of a prime system; if it were, (1) would possess solutions (A.D.E., §65). Thus there must exist, for some $j \leqq q$, an identity

$$
J_{1}^{\mu_{1}} \cdots J_{j-1}^{\mu_{j-1}}\left(S A_{j}-H_{1} H_{2}\right)-L_{1} A_{1}-\cdots-L_{j-1} A_{j-1}=0,
$$

where $J_{i}$ is the initial of $A_{i}, i=1, \cdots, j-1$; and where $H_{1}$ and $H_{2}$ are reduced with respect to $A_{1}, \cdots, A_{j}$. Let $\Lambda_{1}^{(k)}, k=1, \cdots, j+1$, represent the systems $\Sigma+J_{1}, \cdots, \Sigma+J_{j-1}, \Sigma+H_{1}, \Sigma+H_{2}$, respectively. We treat each $\Lambda_{1}^{(k)}$ as (1) was treated. The adjunction of a finite number of forms to any $\Lambda_{1}^{(k)}$ produces a system $\Sigma_{1}^{(k)}$, with no solutions, and with basic sets lower than (2) which furnish zero remainders for the forms in $\Sigma_{1}^{(k)}$.

Let us suppose that each $\Sigma_{1}^{(k)}$ contains an element of $\mathcal{F}$ different from zero. We see on examining these systems that there exist relations, procurable by constructive methods,

$$
\begin{aligned}
& 1=P+M_{0} H_{1}+M_{1} H_{1}^{\prime}+\cdots, \\
& 1=Q+N_{0} H_{2}+N_{1} H_{2}^{\prime}+\cdots, \\
& 1=R_{i}+S_{i 0} J_{i}+S_{i 1} J_{i}^{\prime}+\cdots, \quad i=1, \cdots, j-1,
\end{aligned}
$$

accents indicating differentiation, where $P, Q$, and the $R_{i}$ are linear in the $F_{i}$ and their derivatives.

We equate to unity the product of the right-hand members of (4), (5), and the equations (6). If both sides of the resulting equation are raised to a sufficiently high power, determinable in advance, we secure, as Raudenbush has shown, a relation of the type

$$
1=L+T_{0} V+T_{1} V^{\prime}+\cdots \text {, }
$$

accents indicating differentiation, where $L$ is linear in the $F_{i}$ and their derivatives, and $V=J_{1}^{\mu_{1}} \cdots J_{j-1}^{\mu_{j-1}} H_{1} H_{2} \cdot{ }^{3}$ From (3) we see that $V$ can be obtained as a linear expression in the $F_{i}$ and their derivatives. We thus have such an expression as we are seeking for unity.

If, on the other hand, some system $\Sigma_{1}^{(1)}$ does not contain a nonzero element in $\mathcal{F}$, we apply to it the entire process applied to $\Sigma$. We form in this way systems with basic sets lower than those of $\Sigma_{1}^{(j)}$. The systems thus formed for the various $\Sigma_{1}^{(k)}$ receiving our present treatment will be called, with no attempt to describe their complete history, systems $\Sigma_{2}$. In each $\Sigma_{2}$ a basic set yields only zero remainders.

${ }^{3}$ Ritt; J. F., Algebraic aspects of the theory of differential equations, Semicentennial Publications of the American Mathematical Society, vol. 2, p. 44. 
Let us suppose that each $\Sigma_{2}$ contains a nonzero element in $\mathcal{F}$. What precedes shows that, for each $\Sigma_{1}^{(j)}$ as above, unity is linear in the forms of $\Sigma_{1}^{(j)}$ and their derivatives; this, again, gives the expression which we are seeking for unity.

If there are $\Sigma_{2}$ which contain no nonzero element in $\mathcal{F}$, we give them the treatment which is now familiar. By $\$ 67$ of A.D.E., we know that our process can continue for only a finite number of steps, so that the possibility of determining for unity an expression of the type desired is established.

Columbia University 